\title{
Sporadic and familial varieties of tonic seizures ${ }^{1}$
}

\author{
JAMES W. LANCE \\ From the Department of Neurology, Prince Henry Hospital, Sydney, and \\ the School of Medicine, University of New South Wales
}

The term 'tonic seizure' implies the involuntary assumption of an abnormal posture for a limited time without associated clonic movements. One form of tonic seizure is familiar to the clinician and its mechanism is fairly well understood, the 'tonic cerebellar fit'. The attitude of the body during such a fit is one of extension of the neck, arms, and legs, with pronation of the forearm, flexion of wrists and fingers, and plantar flexion and inversion of the feet. This resembles the decerebrate posture of the experimental animal, and is considered to be caused similarly by any lesion which periodically produces a functional transection of the midbrain (Kinnier Wilson, 1920). However, there is another type of tonic cerebellar fit which was described by Hughlings Jackson (1906) in a patient with a tumour of the cerebellum, in which the posture differed from that of decerebrate rigidity by the upper limbs being in flexion, a feature which led Jackson to call the fits 'tetanus-like'. Consciousness may be retained in such attacks.

In 1924 Sterling reported a series of patients who suffered from transient painful spasms of the face, neck, and limbs in the course of encephalitis lethargica, using the adjective 'tetanoid' to describe the postures of hands and feet. The term 'extrapyramidal epilepsy' applied to these attacks by Sterling is not really appropriate, since some of the spasms lasted as long as six hours, and their mechanism was, and still is, unknown. Wimmer (1925) recorded the case of a boy who developed attacks of torticollis with unilateral tonic spasm of the limbs in extension. Five years after the start of the attacks disorder of movement became generalized and continuous with the appearance of athetosis and torsion spasm. Wimmer regarded the initial tonic spasms as being an epilepsy originating in the corpus striatum. The histories of two patients with brief tonic spasms were reported by Spiller (1927) under the title of 'subcortical epilepsy'. One of these patients, whose spasms involved a lower limb, had clinical evidence of multiple lesions in the optic nerves and spinal cord. The other suffered from unilateral carpopedal

${ }^{1}$ This work was supported, in part, by a grant from Ciba Co. (Pty.) Ltd. spasms in association with a progressive hemiplegia and involvement of the ocular sympathetic supply on the same side. No necropsy was performed.

Kinnier Wilson (1930) related the history of a boy aged 5 who developed a mild left choreo-athetosis, and shortly afterwards became subject to transient attacks of left-sided spasm. The boy was unable to speak during the attacks, in which the arm flexed at the elbow, the fingers flexed, the leg extended, and the foot inverted for up to three minutes. The attacks were precipitated by a knock, fright, or excitement. After six years a permanent rigidity of the face and the left leg became apparent.

Since 1930 the subject of 'striatal' or 'extrapyramidal' epilepsy seems to have been neglected in the English medical literature, although similar unilateral tonic spasms have been reported as a central manifestation of hypocalcaemic tetany (Simpson, 1952) and as a symptom of multiple sclerosis (Matthews, 1958; Joynt and Green, 1962). In the spasms described by Matthews the hand assumed the accoucheur position in three out of four cases, but the fingers were fully flexed into the palm in the fourth. The leg was involved in three patients and was extended at the knee with the foot inverted and plantar flexed and toes were flexed. The spasms were painful in three of the four patients, and the fourth felt a tickling sensation in the affected hand. The patients of Joynt and Green suffered similar attacks but it is noteworthy that their fingers became fully flexed and in only one of four cases were the spasms painful. A recent paper by Lishman, Symonds, Whitty, and Willison (1962) described seizures induced by movement, and six of their seven patients were subject to tonic spasms while remaining conscious. The attacks in the seventh patient consisted of a transient athetosis, and two of the other patients were seen to have athetoid movements at the time of the spasm. Abnormal sensations of numbness, vibration, and tautness, but not pain, were noted in the affected limbs before the attacks. There was no evidence of fixed or progressive neurological deficit.

It is therefore apparent that tonic seizures without loss of consciousness may appear as a symptom of 
many neurological disorders. The common denominator, i.e., the physiological mechanism disrupted to produce a given abnormal posture, may prove eventually to be deducible from a study of a large number of patients varying in source and nature of neurological damage.

Eight patients are presented here to demonstrate clinical variations of this unusual condition. Their case histories divide naturally into two groups: those in which tonic spasm occurs in the course of a recognized neurological disease, and those in which the nature of the underlying disorder is obscure. Five of the patients in the latter group belong to a unique family who had regarded their attacks as stemming from the senior member's experiences in the first world war because the condition had then bsen diagnosed as 'shell-shock'. This hereditary 'shell-shock' is now in its third generation.

\section{TONIC SEIZURES ASSOCIATED WITH OVERT NEUROLOGICAL DISEASE}

CHOREO-ATHETOSIS Case 1 A young woman aged 19 has had right-sided choreo-athetosis since the age of 12 months. Attacks of painful right-sided tonic spasm began when she was 12 years old.

There is no family history of relevant neurological disorder. The patient's birth was assisted with forceps, but she appeared to be a normal baby until she started to walk at the age of 12 months. She stood on the toes of the right foot, with the right knee flexed, and walked with a limp. She preferred her left hand to her right. Occasional twitching and writhing movements of her right hand were noticed soon afterwards, and these have persisted.

At the age of 12, she slipped while running upstairs, and immediately became conscious of a pain over the right half of the body. The right arm then flexed and the right leg stiffened for about 10 seconds.

Since that time she has been subject to similar attacks and has had as many as six in a day. She has had eight nocturnal bouts lasting for two hours, during which attacks of tonic spasm have recurred at intervals of two to three minutes. With the exception of these severe bouts at night, the attacks have occurred most commonly in the mornings between 9 a.m. and noon. They have often been precipitated by a sudden fright or surprise, but usually arise spontaneously and last from 10 to 30 seconds.

A typical attack starts with a sudden pricking feeling in some part of the body as though she had been jabbed with a needle. There is no consistency about the site of this initial sensation. A diffuse pain then spreads over the entire body, more severe over the right side. She is unable to move the right side of the body, but observes that the fingers and thumb of the right hand flex at the metacarpophalangeal and interphalangeal joints, and the right arm flexes at the elbow so that the hand moves toward the chin. The right leg becomes stiff, but she is not aware of any flexion or inversion of the foot. The right side of the face is pulled up. She can hear what is being said to her, and can talk without much distortion of speech. She has not tried to chew during an attack, but food stays in her mouth and she feels unable to swallow.

No change has been noticed in the pupils or in her colour. She does not sweat.

The whole of the right side of the body feels 'tight', but she does not experience numbness or tingling. She is able 5 to walk while the spasm is in progress but 'the whole side $\$$ has to move together'. Consciousness has never been $\overline{\bar{D}}$ impaired, and the sphincters are undisturbed. No clonic movements have appeared at any time. Her general health is good apart from a capricious appetite. She is of normal $\overline{\bar{u}}$ intelligence and is employed as a secretary.

The patient is an attractive young woman who walks with a limp. The fundi and cranial nerves were normal to examination, and there was no pigmentation of the cornea. The right arm and leg were shorter than the left. The $\triangle$ right wrist was held flexed and she was unable to extend it voluntarily. The fingers are usually flexed but she can extend them sharply, when they assume a $\vec{\circ}$ hyperextended posture. While the arm was held out the wrist underwent slow pronation and supination, $\vec{\omega}$ with occasional jerks of flexors or extensors. Stretch reflexes were increased in the flexors of the right wrist and fingers. There was weakness of hip and knee flexion on the right, and movements of the right foot were $\sigma$ limited to a few degrees of dorsi- and plantar-flexione There was no inversion deformity. The toes abducted o when any movement of the foot was attempted. R\& petitive movements were slow and clumsy on the righ side of the body. The tendon reflexes were brisker of 7 the right side and the right plantar response was $\mathbb{D}$ tensor. Surprisingly, the abdominal reflexes were bris and symmetrical.

Sensation was intact and general examination shower no abnormality.

A routine E.E.G. showed some 5-7 c/s activity over the posterior aspect of the left hemisphere but there were no paroxysmal changes. No other tests were done as the patient was on a brief visit from another State.

The patient had been free of attacks for one year while on Dilantin (phenytoin), $200 \mathrm{mg}$. three times daily. Administration of Dilantin was then stopped and the $Q$ attacks recurred. She did not respond to the resumption $\overrightarrow{\vec{F}}$ of the same dosage, and phenobarbitone, $120 \mathrm{mg}$./day, was added to her medication without result. Her treatment was changed to Mysoline (primidone), $500 \mathrm{mg}$. t.d.s., four months ago, and she has had no further attacks. MULTIPLE SCLEROSIS Case 2 A trained nurse aged 47 had suffered an attack of right-sided facial paraesthesiae $\overline{0}$ 15 years ago, two episodes of diplopia 12 and 10 years ago, and a right retrobulbar neuritis two years ago. Each of 3 these illnesses lasted about three weeks. Four months ago she had three tonic seizures with painful flexor spasm of the left arm and leg which was sustained for about five minutes. Her left leg has felt unreal to her since the sei- 음 zures.

The patient has a family history of hypertension, tut not of neurological disorder.

Fifteen years ago she experienced numbness and tingling of the right upper lip and cheek, which lasted for $N$ several weeks. During this time, touching this part of the $N$ face, or the act of eating, would initiate a severe aching. 
pain over the area. Twelve years ago she suddenly developed diplopia, worse on looking to the right. This cleared up after three weeks, but a similar episode occurred two years later, again lasting about three weeks, and she was left with a tendency to double vision on full deviation of the eyes to either side.

Eighteen months ago, vision in her right eye suddenly became blurred. When she closed the left eye she could see only the lower part of the field with her right eye. This resolved completely after about three weeks.

She remained well until four months ago, when she was awakened at 1 a.m. by a severe cramping pain in the left arm and leg. The toes of the left foot flexed and the foot plantar-flexed and inverted. The whole of the left lower limb became rigid in a posture of slight flexion at the hip and knee. At the same moment the fingers and thumb of the left hand flexed partly as though the patient were holding a small ball. The left elbow flexed completely, and the shoulder adducted. The patient's mother and sister were called, and tried to extend the elbow forcibly so as to relieve the 'cramp', but were unable to do so as the muscles were in such strong contraction. The face was not affected and she was able to move her eyes, speak, and swallow. The painful spasm lasted about five minutes and then resolved in a matter of seconds. A feeling of numbness, tingling, and heaviness remained in the left leg ('a sort of deep numbness as though the leg does not belong to me'), and this sensation of unreality has persisted until the present.

She suffered two similar attacks in the next few weeks. She was standing as one spasm developed and the left leg withdrew about 3 inches from the ground, so that she was compelled to hold on to a post to prevent herself from falling. The left arm flexed as before. Each spasm was intensely painful, and lasted two minutes on one occasion and about five minutes on the other.

She has had no further attacks, and the only residual symptom is the sensory disturbance involving the left leg.

On examination, the fundi oculorum appeared normal apart from slight narrowing of the arterioles with arteriovenous nipping. The optic discs were not pale. She experienced diplopia on lateral deviation in either direction. There was no nystagmus and the remainder of the cranial nerves were normal. No other abnormality was found on full examination of the nervous system. Sensation in the left lower limb was normal to touch, pinprick, vibration, proprioception, two-point discrimination and figure-writing. Extinction phenomena could not be demonstrated. General examination was normal with a blood pressure of $140 / 185 \mathrm{~mm}$. $\mathrm{Hg}$.

A routine E.E.G. and radiograph of the skull and chest were nornal. The serum calcium level was $10 \mathrm{mg} . \%$.

\section{TONIC SEIZURES WITHOUT OTHER INDICATIONS OF} NEUROLOGICAL DISEASE

SPORADIC Case 3 A medical student aged 21 has been prone to attacks of left-sided tonic spasm since the age of 13 years.

The patient's birth and childhood were normal with no history of convulsions or migraine. A sister died of some infection a few weeks after birth, and there are no other siblings. His father has signs of an intramedullary lesion of the cervical cord as yet undiagnosed, but there is no history of epilepsy or related condition among his forebears. He has always been rather tense and nervous with a tidy, meticulous personality.

The attack starts with a 'nervous sensation' in the epigastrium, then five to 10 seconds later he feels his tongue twist inside the mouth, his jaws clench, his face feels rigid, and he is unable to smile. He can select the words he requires, but he cannot speak normally, and he makes sounds which are 'thick and odd'. His gaze is fixed but there is no diplopia. One attack came on while playing tennis and his eyes were unable to follow the ball. No comment has been made about the size of his pupils. He has not tried to swallow during an attack but feels that he could not. He has not noticed excessive salivation.

The fingers of his left hand extend and partly abduct (dropping any object held in the hand), then flex at the metacarpophalangeal joints and slightly at the interphalangeal joints. The thumb adducts but the digits do not touch one another. The left elbow then slowly flexes to about 90 degrees. The left leg becomes rigid with slight flexion at the hip and knee and adduction of the hip; at the same time the left foot inverts. He is quite unable to initiate movement at the ankle joint but can continue to walk, or even to run, with an unwieldy, stumbling gait. If he attempts to extend the left elbow and wrist by manipulation with his normal right hand, he feels a resistance to the movement which increases as the movement takes place and he is unable to restore the limb completely to its resting posture. His chin turns towards the right, the occiput tilts to the left, and the left shoulder draws up toward the back of the head. There are no paraesthesiae, sphincter disturbances, or headache.

The entire attack lasts for less than a minute-sometimes only a few seconds. It is always precipitated by a situation involving startle, self-awareness, or embarrassment, e.g., giving a speech or demonstration, answering the telephone or taking part in some game when spectators are present. The attacks are not preceded by or associated with overbreathing. He can sometimes prevent an attack by grasping some object firmly in his left hand and taking a slow deep breath. The frequency of attacks may be only once in four weeks when he is relaxed on vacation, but may be up to six times a day immediately before university examinations.

On examination, the left forearm was about $5 \mathrm{~mm}$. shorter than the right, but no other abnormality was found in the nervous or other systems. There was no pigmentation in the cornea. A radiograph of the skull, a right common carotid arteriogram, and a pneumoencephalogram were completely normal. His E.E.G. was normal and remained so after the intravenous injection of $10 \mathrm{ml}$. Metrazol $5 \%$, a dosage sufficient to cause twitching of the muscles and myoclonic jerking in response to sudden noise. During voluntary hyperventilation he commented on the sensation of 'pins and needles' but there were no abnormal movements, and he stated afterwards that the sensation was completely different from that of his attacks. The E.E.G. record 
demonstrated 3-7 c/s waves bilaterally on overbreathing but no paroxysmal changes developed.

The serum calcium level was $9.8 \mathrm{mg} . \%$, and other electrolytes were also within the normal range. The cerebrospinal fluid was completely normal.

He has not adhered to any anticonvulsant régime long enough to assess its efficacy.

FAMILIAL Cases 4-8 are of this category.

Case 4 A man aged 68 (II.2 in the family tree illustrated in Fig. 1) has been subject to unilateral or bilateral attacks of tonic flexor spasm since the age of 22 .

The patient has always been a tense person who speaks and acts quickly, and is liable to dull occipital headache at times of stress. He suffered wounds to the right arm and jaw in the First World War, but has had no illness in the past other than the attacks to be described.

The attacks come on when he is relaxed, usually in the evenings, but at times during the lunch hour at work. Emotional upsets or a sudden fright have evoked an attack on occasions. It is rare for a day to pass without an episode although he has had some periods of freedom of one or two weeks.

His wife reports that his eyes 'go glassy' at the start of an attack, and one side of the face draws up. One or other arm then flexes so that the fist presses firmly into his neck, and the leg on the same side lifts off the ground but does not flex completely onto the abdomen. If he is not assisted, he will topple onto the floor. As soon as he lies down he goes to sleep, the limbs relax, and he usually awakens in five to 30 minutes feeling well. If he is awakened prematurely, the attack will start again and if he is startled it may go on for an hour. The arm and leg involved are always on the same side of the body, more commonly right than left, but at times both arms and legs flex.

The only subjective sensations he experiences are a general feeling of nervousness and sometimes 'pins and needles' in the affected legs at the start of an attack. All observers are sure that he does not overbreathe. He can hear but is unable to speak while the attack lasts. One attack was so severe that he fell to the ground and was unable to make any movement other than blinking his eyes.

The nervous and other systems were normal. Unfortunately limb length was not measured.

A radiograph of the skull and chest and an E.E.G. showed nothing of significance. The blood Wassermann reaction was negative. He declined admission to hospital for further investigation.

Case 5 A man aged 41 (III.4 in Fig. 1) has had attacks of unilateral tonic flexor spasm, affecting either side, from the age of 2 years.

Shortly after he began to walk the patient was seen to have his first episode of involuntary movement, spasm, and 'paralysis'. During his childhood attacks were infrequent, perhaps one every few months, but never at intervals longer than six months. They were always brought about by emotional upset or the approach of some excitement such as a birthday party. In adult life he has had bouts of a month in which episodes occurred every other day, usually after working particularly hard.

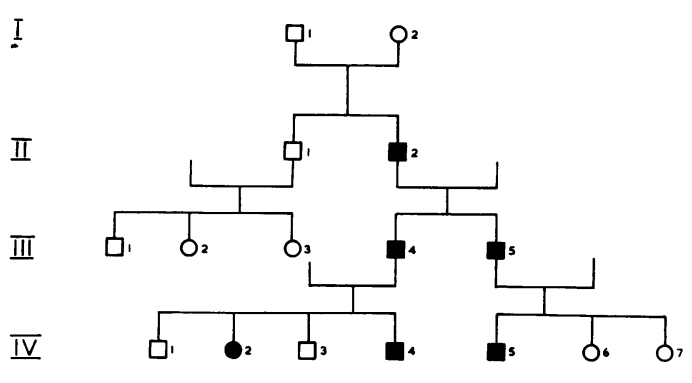

FIG. 1. Family tree of cases 4 to 8 .

The attack commonly comes on while he is relaxing after being in a state of tension, excitement, or exhaustion, $\leadsto$ particularly if he takes an alcoholic drink. He points out $\vec{O}$ that the tendency to produce attacks does not vary as to the amount of alcohol ingested, but is closely linked to his $\vec{\omega}$ mental state at the time. If he is at a party where he has some responsibility for attending to the needs of other guests, he is able to drink a considerable amount without inducing an attack. On the other hand, a casual drink at a $?$ bar with friends almost invariably provokes an attacko The attacks may come on at any time of the day or nigh $\bar{G}$ and last from five to 50 minutes, developing and passing 응 off gradually. If he feels an attack starting, he can some times 'fight it off' by physical activity, forcing the $\lim B$ to do his bidding. Once an attack is established it resolve more rapidly if he lies down in a quiet room, relax 8 completely and takes slow deep breaths. He is quite certain that the rate and depth of his respiration is not increased during the onset or at the height of his attacks $\overrightarrow{5}$

The pattern of the illness is constant. The muscular $\omega$ spasm is always unilateral but the side varies in different episodes. He first notices the fingers of one hand closing to make a fist. The thumb flexes and may be trapped inside the closed fingers or remain outside. The elbow then flexes and the arm rotates internally so that the forearm rests across the chest in a Napoleonic attitude. At the same $₫$ time the toes flex, the foot plantar-flexes and the knee and $\overline{\bar{z}}$ hip flex so that his foot is lifted off the ground. In the early stages he can overcome this movement with difficulty by voluntary exertion but the limb soon flexes again. The opposite side of the body maintains a normal posture but feels weak and movements are slow. If he attempts to restore the position of the contracted limb to normal, resistance in the flexors is marked; 'the muscles are in a real spasm'. Any movement of the contralateral side appears to aggravate the spasm on the affected side. He has never fallen in an attack as five to 10 minutes elapse between the onset and the height of the spasm, so that he is able to lie down 'and make the bed first if necessary' before being incapacitated.

His voice becomes monotonous and blurred, although 을 he selects the correct words. As the disability deepens, his $\mathrm{N}$ expression is limited to grunts, and finally, in a severe or attack, he is completely mute. He can see and hear $N$ perfectly, and his mind is 'crystal clear'. He can move the $\mathrm{N}$ eyes but not the head or neck. His lower face becomes 
fixed so that no expression is possible, but he is able to close the eyes and blink. His jaw does not appear to be clenched as the mouth is usually slightly open and saliva dribbles from the mouth. He is unable to swallow, cough, or protrude the tongue. There are no abnormal sensations in the face or body. He feels hot but there is no change in colour. Sweating has been noticed in the palm of the clenched hand. $\mathrm{He}$ is able to pass urine normally while the attack is in progress.

The attack takes 10 to 15 minutes to resolve, and he then feels well without headache or 'hangover'.

There is no history of clonic movement or convulsion. $\mathrm{He}$ has lost consciousness only once in his life, and this followed a feeling of faintness when he was suffering severe colicky abdominal pain as a result of 'food poisoning'.

$\mathrm{He}$ works as a crane-driver and is perfectly well between attacks.

On examination, the fundi oculorum and cranial nerves were normal although the palpebral fissures were narrow. The larynx was prominent but the sternomastoid and other muscles were strong and there was no myotonia. There were crescents of arcus senilis in the corneae but no Kaiser-Fleischer rings. Full examination of the nervous and other systems showed nothing of significance apart from the left forearm being $6 \mathrm{~mm}$. shorter than the right. He is right handed.

A radiograph of the skull and chest and an electroencephalogram were normal. He was not prepared to submit to further investigation.

Case 6 A girl aged 11 (IV.2 in Fig. 1) has been subject to unilateral tonic flexor spasms affecting either side since the age of 2 .

The patient was born after a long and difficult labour, but appeared to be a normal baby, and progressed well.

Her attacks started when she was 2 , and closely resemble those of her father (case 5), although shorter and less severe. They used to occur about once every three months as a young child, but in recent months she has been having attacks after her evening meal almost every night. Her speech becomes slurred, then her arm and leg on one or other side flex. Her face is immobile, but no change has been noticed in eye movements, pupils, or colour. She cannot speak, swallow, or cough. She is put to bed and soon goes to sleep. The attack has never lasted longer than 10 minutes before sleep supervenes. On occasions she has had mild attacks which pass off after lying down for two to five minutes.

The attacks are brought on by physical tiredness, after running and playing all day, or by excitement, e.g., setting off for a holiday. The child cannot add much to her parents' description. 'The arm comes up and is kind of paralysed. I can't get it down and can't talk or move my leg'. The attacks are always unilateral, left more than right.

The child is a plump girl of healthy appearance. Apart from the left forearm being $6 \mathrm{~mm}$. shorter than the right, no abnormality was noted on full physical examination. She is right handed.

A radiograph of the skull and chest were normal. A routine electroencephalogram showed excessive slow activity for her age with marked instability on over- breathing, but there were no focal or paroxysmal changes.

Case 7 A boy aged 8 (IV. 4 in Fig. 1) has had attacks of unilateral or bilateral tonic flexor spasm from the age of 2 years.

The patient's birth was normal. Since the age of 2 he has had attacks which are similar to those of his father (case 5) but milder. The frequency of attacks varies from two a week to one in three months, being greater when he is exhausted or excited. They commonly occur at bedtime.

Most attacks are unilateral, right more than left, but he has had some episodes in which both sides have been similarly affected.

The toes and foot curl downwards, then the leg flexes at the knee and hip. He tries to push the leg down but cannot. His hand clenches and he is unable to open it, and he becomes completely mute. If the attack is mild he may be able to move about and ward it off. More commonly he is put to bed and goes to sleep after about 10 minutes. He states that he is able to move his face to some extent during the attack, and is able to swallow.

The patient is a bright, alert child. The only abnormality noted on full physical examination was that the left forearm was $4 \mathrm{~mm}$. shorter than the right.

A radiograph of the skull and chest were completely normal. A routine E.E.G. was within normal limits for his age apart from one burst of high voltage $4 \mathrm{c} / \mathrm{s}$ waves which occurred bilaterally on eye closure.

Case 8 A boy, now aged 14 (IV. 5 in Fig. 1), has suffered from 'turns' since the age of 3 , and bilateral tonic spasm from the age of 9 years.

The patient's birth was prolonged, but early milestones were normal and he has not had any other serious illness. Between attacks he is an active boy who plays many sports without undue fatigue.

At the age of 3 he had an attack in which he suddenly stopped moving, his eyes looked strange, and he appeared flushed for about 10 minutes. There were no movements of the limbs. He had further attacks of a similar nature at intervals of approximately six months.

At the age of 9, the attacks became more complex, and the pattern has remained constant since then. He first notices an unpleasant, peculiar sensation in the face and throat, and he tries to clear the throat by little coughs. This lasts for about 10 minutes, during which time he is restless and moves the limbs about. He then becomes aware that the right arm feels stiff, the fingers of the right hand flex partly into the palm and the thumb flexes to meet the index finger. The elbow slowly flexes across the chest. On rare occasions, the fingers of the left hand have been involved as well, but the left elbow has never flexed. His mother can easily overcome the flexion of fingers and elbow by manipulation, but the limb resumes its position when she releases her grip. At the same time as the upper limb is affected, the toes of the left foot "curl under the foot'. The right lower limb has never been affected. If he is lying in bed when the attacks start he finds it hard to initiate movement and has to push himself up slowly on to his elbow. Although he feels 'stiff and tight' all over, he is able to move all limbs and can walk during an attack, limping because of the contraction in his left foot. If he lies down quietly the arm and leg tend to relax, but a 
slight movement will make them flex again. His face is weak, and he is unable to smile as 'the mouth only goes sideways'. On one occasion he attempted to brush his teeth and could not retract the upper lip. He can chew slowly. He is able to speak and to select the correct words, but his voice is slurred and nasal in quality, so that it is often unintelligible. Swallowing is slow and difficult, and he finds that saliva dribbles from the corner of his mouth. Respiration is normal, although he feels a sense of pressure on his chest and is unable to cough. His pupils seem larger than normal to his mother, but his vision and eye movement are unaffected. He can protrude the tongue. The attack is not painful and there are no paraesthesiae. He feels hot and his mother has observed that his face is flushed but he does not sweat. He is able to pass urine during an attack.

The episode develops over a period of five minutes, lasts from 10 to 60 minutes, and passes off rapidly in about a minute. After the first few attacks he was left with a bilateral headache which lasted up to two hours, but more recently he has felt well and is very hungry after recovering from the seizure. He has never lost consciousness in an attack, and has not had any jerks or clonic movements. All attacks except two have been at night and have usually been preceded by fatigue and excitement. The frequency has ranged from two a month to one in six months.

On examination, the only notable feature is his facial appearance. There is a mild bilateral ptosis and the face lacks expression at rest. However, the face undergoes a full range of movement on formal testing and fatiguability could not be demonstrated. Careful physical examination on many occasions has not disclosed any abnormality of the nervous or other systems. There are no Kaiser-Fleischer rings in the corneae. The forearms are of equal length.

Radiographs of the skull and chest were normal and serum electrolytes, including calcium and phosphorus, were normal on two occasions. His E.E.G. has been recorded at the ages of 9,10 , and 14 , and has always shown excessive slow activity $(5-7 \mathrm{c} / \mathrm{s})$ without focal or paroxysmal features. After the intravenous injection of $150 \mathrm{mg}$. Metrazol in two minutes, repeated paroxysms of high voltage 3-4 c/s spike and wave appeared in the record.

The pneumoencephalogram displayed an unusual feature. There was a constant filling defect about $1 \mathrm{~cm}$. long and $0.5 \mathrm{~cm}$. deep near the midpoint of the superior margin of the body of the left lateral ventricle. However, a left carotid angiogram, which clearly demonstrated both anterior cerebral arteries, was normal.

He continued to have attacks while taking Dilantin, $100 \mathrm{mg}$. three times daily, and Prominal, $60 \mathrm{mg}$. three times daily. The latter was changed to Mysoline, $250 \mathrm{mg}$. three times daily, three years ago with reduction in the frequency of the seizures. He has not had an attack for the past year while on medication.

FAMILY HISTORY OF CASES 4-8 In generation I, the father of case 4 died of war wounds, and the mother died at the age of 40 , cause unknown. Neither are known to have had any form of seizure.

Generation III contains the two sons of case 4, the younger of whom (III. 5) was the father of case 8, and $Z$ died of ulcerative colitis at the age of 37 . His widow does $\stackrel{\mathbb{D}}{\stackrel{D}{-}}$ not know the age at which his attacks started, but she witnessed four attacks in the last eight years of his life in which his right hand flexed for 10 to 20 minutes, and inc one episode both legs also flexed. The attacks occurred 0 particularly with the combination of alcoholic intake and excitement. His face was not affected, and he could speak ? and swallow during the attack. These seizures were not mentioned in the record of his final admission to hospital, .: and the necropsy report states that no macroscopic $\overrightarrow{\vec{F}}$ evidence of abnormality was found in the brain.

\section{DISCUSSION}

The tonic seizures described in this paper show the $\frac{\bar{\Phi}}{\frac{\sigma}{\alpha}}$ following clinical features:-

DISTRIBUTION Muscular spasm was unilateral in $\vec{\circ}$ the first three patients and bilateral in the familial cases.

DURATION The seizure lasted less than one minute in cases 1 and 3, two to five minutes in case 2 , and five to 60 minutes in the familial cases.

SENSORY PHENOMENA The seizures were intense painful in the first two patients, but completer painless in the others, although the degree of mus cular spasm appears to have been comparable. Case $\mathbb{D}$ 1 commented on a sudden pricking sensation in some part of the body which immediately preceded the diffuse severe pain. The second patient had noticed sensation of the affected limb 'not belonging to hep since the seizures, although no sensory loss could be demonstrated by clinical testing. The third patient was aware of an epigastric aura before the seizure began. The fourth patient experienced pins and needles in the affected leg at the start of an attack, and the eighth felt an indescribable unpleasant sensation in the face and throat. All patients commented on sensations of stiffness and tightness in the muscles.

CRANIAL NERVE FUNCTIONS All patients, except the second, noticed involvement of muscles innervated by the cranial nerves. The face was described as being pulled up on one side by the first patient, and as rigid, fixed, weak, or immobile by the remainder. Most developed slurred speech and four out of the five familial patients have become completely mute. Chewing, swallowing, and coughing became difficult or impossible. One patient (case 3) stated that his 온 tongue seems to turn sideways in his mouth. In the $>$ familial cases, the symptoms of a severe attack appear to be those of a reversible pseudobulbar palsy. N POSTURE OF THE LIMBS In all patients the upper limb was adducted and internally rotated at the shoulder, N 
and flexed at the elbow, wrist, metacarpo-phalangeal and interphalangeal joints. Attempts by observers to straighten the limb met with active resistance from the contracting muscles.

The most constant feature about the posture of the lower limbs was plantar-flexion and inversion of the foot on the affected side. The leg became rigid and six of the eight patients described flexion at the hip and knee joints so that the leg was lifted from the ground if they were standing.

The posture of the upper limbs thus resembles that of a hemiplegic patient or that of Jackson's tetanuslike seizures rather than that of hypocalcaemic tetany. The posture of the lower limbs is one of rigidity in partial flexion, with the exception of the feet which adopt the equinovarus position common to many dystonic states.

PHYSICAL SIGNS The first patient presented the signs of right-sided choreo-athetosis with shortening of the limbs on the right side, and her history is remarkably similar to that of Kinnier Wilson's patient mentioned in the introduction to this paper. The second patient, who suffers from multiple sclerosis, had diplopia at the time of examination, but no other abnormality could be found. In the remaining patients, the only physical sign which might possibly be of significance was a minor asymmetry of the forearms noted in four of the six, the left being about $5 \mathrm{~mm}$. shorter than the right. Such asymmetry is often indicative of a lesion dating from birth and involving the post-central cortex (Penfield and Robertson, 1943).

INVESTIGATIONS The E.E.G. record showed no specific abnormality in any patient. Metrazol activation was employed in two patients but did not produce a seizure. Wave-and-spike paroxysms appeared in case 8 after $150 \mathrm{mg}$. Metrazol, but, as such paroxysms may be found in one third of normal subjects under the age of $\mathbf{3 0}$ given Metrazol (Moore, Kellaway, and Kagawa, 1954), no importance is attached to this finding. Unfortunately the opportunity to make a recording during a seizure did not arise.

Matthews (1958) reported that the amplitude of fast activity increased as a tonic seizure started before the tracing was obscured by muscle artefact.

No significant E.E.G. change was noted by Biagio and Spaccarelli (1959) during a tonic seizure. Kennedy (1959) described a short burst of low voltage fast activity, followed by bilateral suppression of cerebral rhythms, then recurrence of fast activity and rhythmic 6-7 c/s waves. Joynt and Green did not find any alteration in the E.E.G. tracing during tonic seizures in two of their patients with multiple sclerosis.
Arteriography and pneumoencephalography were carried out on two patients. One of these (case 8) demonstrated a small filling defect in the roof of the left lateral ventricle. Every effort was made to induce other members of the affected family to undergo similar investigations without success. The significance of this isolated finding is therefore hard to assess, but it directs attention to the possibility of a congenital lesion in the region of the supplementary motor area, which will be discussed later.

\section{THE MECHANISM OF TONIC SEIZURES}

It must be agreed that case reports of tonic seizures 'form very unpromising material for any attempt to determine the site of the responsible central lesion or the nature of the central mechanism involved' (Matthews, 1958). Nevertheless a few general observations can be made on the clinical pattern.

The rapidity of onset and resolution, and brief duration, of the attacks in cases 1-3 is consistent with a paroxysmal neuronal discharge, and the term epilepsy may reasonably be applied. The more leisurely pace of the seizure in the familial cases resembles the phenomena of migraine rather than epilepsy, and suggests the possibility of cerebral vasospasm or 'spreading depression' as the underlying mechanism. However, only one patient (case 8) remarked on headache following an attack.

The site of commencement of the seizure may be indicated by the subjective sensations of some patients. Intense pain preceded and accompanied the muscular spasm in the first two patients, and is a common feature in previous reports of tonic seizures. Other patients of the series experienced equally strong contraction of muscles without pain, hence it is unlikely that stimulation of nerve endings in muscle is sufficient to account for the pain of some seizures, and a central origin must be considered.

One of the patients had a persistent sensation of unreality concerning the mental image of the affected leg after her attacks, suggesting a lesion in the afferent connexions of the parietal association cortex. One patient (case 8) experienced an unpleasant prodromal sensation in the face and throat. Another (case 3) described an epigastric aura. The remaining three patients commented only on the sensation of stiffness in the affected limbs, which may well have been of peripheral origin. It is of interest that Matthews' patients with multiple sclerosis reported pain, a 'peculiar horrid' sensation and a tickling feeling as accompaniments of attacks, and the patients of Lishman et al. (1962) commented on similar prodromal symptoms. It is therefore postulated that some tonic seizures start with abnormal 
activity in the thalamus and specific thalamocortical pathways.

Shortly after or at the same time as the abnormal sensations, the flexed attitude develops and the power of voluntary movement is lost in the affected limbs, implying that the cortical control of postural mechanisms has been functionally interrupted. Denny-Brown (1960) differentiates between two basic postures found as the end-result of damage to the basal ganglia: the 'striatal syndrome' with rigid flexion of the upper limbs and extended lower limbs, and the 'pallidal syndrome' of generalized flexion with akinetic mutism. Both postures have in common plantar-flexion and inversion of the ankles.

The attitude adopted in the tonic seizures described is akin to that of the pallidal syndrome, although the fingers may be extended in the latter. It differs from the striatal syndrome only by slight flexion at hip and ankle in our patients. Certainly one has seen postures close to those of tonic seizures in both hemiplegic and Parkinsonian patients in their final dystonic stage. The seizures thus indicate a short-lived deficiency in function of the basal ganglia, their afferent connexions from the cerebral cortex or their efferent connexions with the reticular formation.

One cannot with certainty label the attacks as 'subcortical' seizures. The supplementary motor cortex is known to exert some control over postural adjustments, producing tonic muscular contraction which is often bilateral (Penfield and Jasper, 1954). The pattern of seizures arising from this area has been analysed by Ajmone Marsan and Ralston (1957) and is most commonly abduction of the contralateral arm with turning of the head toward the elevated hand. An epigastric aura has been noted in $28 \%$ of patients and somatic sensory phenomena in $64 \%$. Speech is affected in $50 \%$ of patients, and consciousness is usually retained in $71 \%$. One side of the body was found to be smaller than the other in $21 \%$ of patients. Auditory startle is particularly effective in precipitating fits originating in the supplementary motor area. There are thus a number of points in common between supplementary motor seizures and the tonic seizures here described. Kennedy (1959) reported the clinical and E.E.G. findings in seven patients who had midline cerebral lesions. Three of these patients had suffered tonic seizures and one adopted a posture of flexion of the right arm and leg in the attacks, which were preceded by an epigastric and genital sensation. At operation, a meningo-cortical scar was removed from the upper part of the central fissure.

It is possible that tonic seizures result from a temporary release of basal gangliar and reticular postural mechanisms from cortical control in the same way as the grasp reflex is released by destruction of the medial frontal cortex.

Until more is known of the mechanism of theen attacks it is advisable to discard the terms 'sub cortical', 'striatal' and 'extrapyramidal' epilepsy, and describe the attacks simply as 'tonic seizures', or, to distinguish them from transient decerebrate rigidity, 'dystonic seizures'.

Most reports in the literature have dealt with. dystonic seizures as a symptom of a known neuro- $\overrightarrow{\vec{p}}$ logical disease, e.g., a sequel to hemiplegia of vascular or traumatic origin, an accompaniment of choreo-athetosis or a manifestation of encephalitis $\overline{\frac{\sigma}{\infty}}$

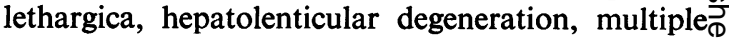
sclerosis, or hypocalcaemic tetany. The first two patients of this series fall into this symptomatic group. क

It is possible that some structural defect is also $\vec{\circ}$ present in the nervous systems of the remainder. The $-\overrightarrow{-}$ features which make one suspect this possibility are $\vec{\omega}$ the asymmetry of the limbs in cases $3,5,6$, and 7 , and? the small filling defect in the roof of the left lateral ventricle in case 8. Case 3 of this series resemblesin closely the patients described by Lishman et al. (1962) who were also young males, but these authors difl $\vec{i}$ not comment on any asymmetry of the limbs ofr possibility of birth injury.

As far as one is aware, this is the first report of t familial occurrence of tonic seizures.

A necropsy was performed on one member of the affected family (III.2) who died of ulcerative colits seven years ago, and the report states that no macre scopic abnormality was found in the brain. Howeve attention had not been directed to the nervous system and it is possible that a small lesion may have been missed. The tendency to dystonic seizures appears to be transmitted by a dominant gene.

\section{SUMMARY}

The case histories of eight patients suffering from a $\overrightarrow{\overline{\vec{O}}}$ form of tonic seizure are reported in two groups, $\vec{J}$ depending on the presence or absence of overt disease of the nervous system. The first group comprises one patient with choreo-athetosis and one with multiple sclerosis. The second group consists of six patients, five of whom belong to a family in which the disorder is apparently transmitted by a dominant gene.

Consciousness is retained during the attacks. The limbs of one or both sides of the body assume a partly flexed posture, with feet plantar-flexed and $\frac{D}{O}$ inverted. The seizures are associated with pain in two instances, and with an epigastric aura, tingling $N$ of the affected leg, and sensations in the face and throat in other patients.

Cranial nerve function is disturbed during the $\omega$ 
seizures in most patients, the condition at the height of the attack resembling pseudo-bulbar palsy, or, in the familial cases, akinetic mutism. The seizures last less than one minute in two patients, two to five minutes in another, and five to 60 minutes in the familial cases.

Slight asymmetry of the limbs was observed in five of the eight patients. A small filling defect was found in the roof of the left lateral ventricle in one of the two patients who agreed to pneumoencephalography being done. No specific abnormality was found in E.E.G.s recorded between seizures.

The mechanism of this form of tonic seizure is at present unknown and the descriptive term 'dystonic seizure' is suggested to distinguish it from transient attacks of decerebrate rigidity.

I wish to thank Miss J. Easthope for her assistance in recording the E.E.G.s in these patients, and the Depart- ment of Medical Illustration, University of N.S.W. Teaching Hospitals, for the figure.

\section{REFERENCES}

Biagio, F. di, and Spaccarelli, G. (1959). Policlinico, Sez. Prat, 66, 830

Denny-Brown, D. (1960). Lancet, 2, 1099.

Jackson, J. Hughlings (1906). Brain, 29, 425.

Joynt, R. J., and Green, D. (1962). Arch. Neurol. (Chic.), 6, 293.

Kennedy, W. A. (1959). Brain, 82, 147

Lishman, W. A., Symonds, C. P., Whitty, C. W. M., and Willison, R. G. (1962). Ibid., 85, 93.

Marsan, C. Ajmone, and Ralston, B. L. (1957). The Epileptic Seizure. Thomas, Springfield, Illinois.

Matthews, W. B. (1958). Brain, 81, 193.

Moore, F. J., Kellaway, P., and Kagawa, N. (1954). Neurology (Minneap.), 4, 325.

Penfield, W., and Jasper, H. (1954). Epilepsy and the Functional Anatomy of the Human Brain. Churchill, London.

—_; and Robertson, J. S. M. (1943). Arch. Neurol. (Chic.), 50, 405. Simpson, J. A. (1952). Brain, 75, 76.

Spiller, W. G. (1927). Ibid., 50. 171.

Sterling, W. (1924). Rev. neurol., 31 (2), 484.

Wilson, S. A. Kinnier (1920). Brain, 43, 220

_ (1930). Brit. med. J., 2, 90.

Wimmer, A. (1925). Rev. neurol., 32 (2), 281. 